\title{
Simulation of the ATLAS New Small Wheel (NSW) System
}

\author{
Koki Maekawa ${ }^{1}$ on behalf of the ATLAS Muon collaboration \\ 1 The University of Tokyo ICEPP, Hongo Bunkyo-ku Tokyo 7-3-1, Japan \\ maekawa@icepp.s.u-tokyo.ac.jp
}

\begin{abstract}
New Small Wheel (NSW) is a major detector upgrade project of the ATLAS muon spectrometer for the LHC luminosity upgrade. NSW comprises sTGC and Micromegas as muon tracking detectors of high rate capability, and front- and back-end electronics for upgrading the muon level-1 trigger. Detailed simulation of the detectors and the electronics has been developed and used for the study of expected performance. An overview of the NSW system and some results of the trigger performance with the Micromegas are presented.
\end{abstract}

Keywords: ATLAS, Muon, New Small Wheel (NSW).

\section{Introduction}

Upgrades of the Large Hadron Collider (LHC) [1] will take place in several phases. After the second long shutdown (LS2) in 2019-20, the LHC instantaneous luminosity will increase up to $3 \times 10^{34} \mathrm{~cm}^{-2} \mathrm{~s}^{-1}$, which is three times the design luminosity, and $7.5 \times 10^{34}$ after the LS3 in 2024-26. At these high luminosities, two problems arise in the ATLAS [2] end-cap region of the muon spectrometer: degradation of muon reconstruction efficiency due to an efficiency drop of the precision tracker in the inner-most muon station (the Small Wheel) at high background conditions, and an increase of fake level-1 muon triggers proportional to the luminosity to an unacceptable rate.

In order to solve these two problems, the Small Wheel will be replaced by a new detector, the New Small Wheel (NSW) [3]. NSW is equipped with tracking detectors of high rate capability $\left(20 \mathrm{kHz} / \mathrm{cm}^{2}\right)$, and a function of generating real-time trigger segments for the use in the level-1 trigger to suppress the fake triggers.

\section{Fake trigger suppression with New Small Wheels}

Current level-1 muon trigger in the end-cap is generated using the trigger segment in the Big Wheels $(\mathrm{BW})$. Transverse momentum $\left(p_{\mathrm{T}}\right)$ of a muon is determined based on the pointing angle of the segment reflecting the bending in the toroidal magnetic field in front of BW (Fig. 1(a)).

Fake Level-1 muon triggers are mainly caused by particles produced in the material before BW. Such fake triggers can be rejected by requiring the presence of a NSW 
(a)

segment in space-time coincidence with the BW segment, reducing the rate of endcap level-1 to about $1 / 3$ [3]. In the NSW trigger processor, trigger segments are reconstructed and a set of parameters, the position of the segment in $(\theta, \phi)$ and the IP pointing angle $\Delta \theta$, are generated (Fig. 1(b)). These information are combined with the BW segment in the Sector logic to produce muon Level-1 signals.

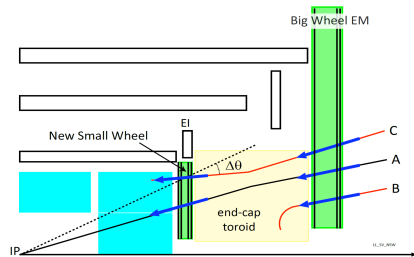

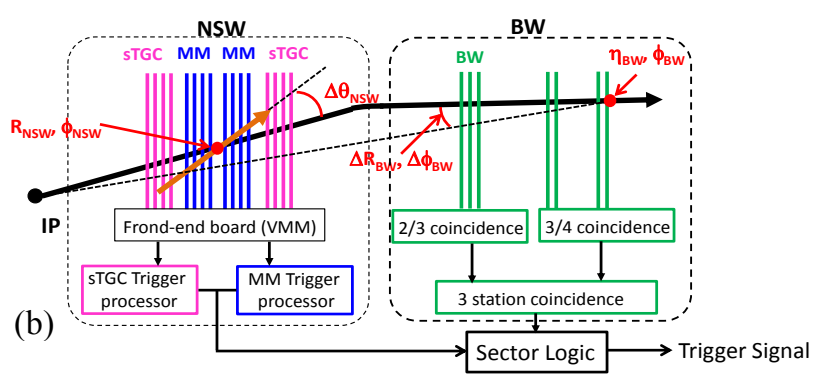

Fig. 1 (a) A $z-y$ view of $1 / 4$ of the ATLAS detector. (b) Trigger scheme with NSW.

(a)

(b)

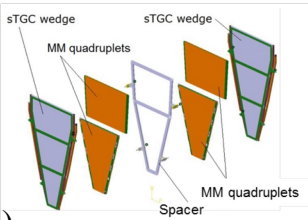

Fig. 2 (a) Overview of NSW. (b) A sector of NSW. (c) MM structure. Drift and avalanche

59 multiplication of ionized electrons are depicted. (c)

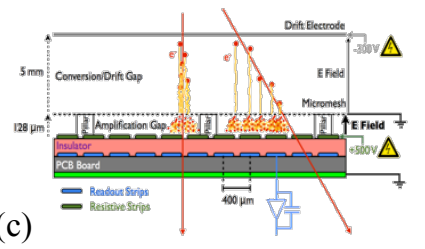


$\begin{array}{lll}60 & 4 & \text { Simulation of Micromegas Trigger System }\end{array}$

61 GEANT4 is used to model the detector materials and geometry as well as the interac62 tion of particles in the detector. The simulation of the MM signals proceeds as fol63 lows. Energy deposits in the sensitive gas volume is used to simulate ionization elec64 tron clusters along the particle tracks. Drift of electrons to the mesh is calculated tak65 ing into account the drift velocity, transverse and longitudinal diffusion, and Lorentz 66 angle using parametrization of GARFIELD calculations. Gas multiplication is mod67 elled according to the Polya distribution.

Pulse shape of amplifier output is modelled according to the characteristics of the amplifier-shaper circuit of the VMM chip. It is then compared to the discriminator threshold to generate hit signals. For each group of 64 channels in a VMM chip, the strip with first arriving hit is identified and the ART signal is generated. Dead time of each input channel after hit signals (300 ns) and of the ART logic after generation of an ART signal (40 ns) is implemented. ART signals from VMM chips are then collected and passed to the trigger processor, where the MM trigger algorithm is performed.

\section{6}

\section{$5 \quad$ Micromegas Trigger Performance Study}

Performance of the MM trigger is studied using simulated single muon events. In order to see the effects of pile-up, inelastic $p p$ interactions are also simulated and added to the single muon event in the same bunch crossing (BC) and several BCs before and after the muon event. The mean number of $p p$ interaction per BC considered here are 80 and 160 , corresponding to the peak luminosity during Run- 3 and HLLHC [4].

\subsection{Segment Finding Efficiency and Trigger Link Occupancy}

Fig. 3(a) shows the timing distribution of the ART signal. In order to collect signals with high efficiency, a time window of $50 \mathrm{~ns}$ (two LHC BCs) or more is needed. In the following, ART signals are collected within $50 \mathrm{~ns}$ time window. The MM detector has 8 layers. 4 of them have strips parallel to $\phi$ direction $(\mathrm{X})$ and the other 4 layers have strips tilted by $+1.5^{\circ}(\mathrm{U})$ or $-1.5^{\circ}(\mathrm{V})$. Fig. 3(b) and (c) show the efficiency of finding trigger segments for different requirements on the number of hit strips (out of $4 \mathrm{X}$ hits and $4 \mathrm{UV}$ hits) found within a common slope $(\mathrm{R} / \mathrm{Z})$ road. The trigger segment finding efficiency decreases at large number of pile-up events due to VMM dead time. However, there is no significant impact for 3X3UV and 2X2UV coincidence threshold. Even at the highest $\eta$ where the background hit rate is the highest, the inefficiency is at most $3 \%$.

Due to bandwidth limitation of data transmission, the NSW trigger electronics can send out at most 8 trigger segments per BC per sector to downstream. The number of tracks due to background particles and fake tracks is less than one with the threshold of 3X3UV and 4X4UV as shown in Fig. 3(d). 

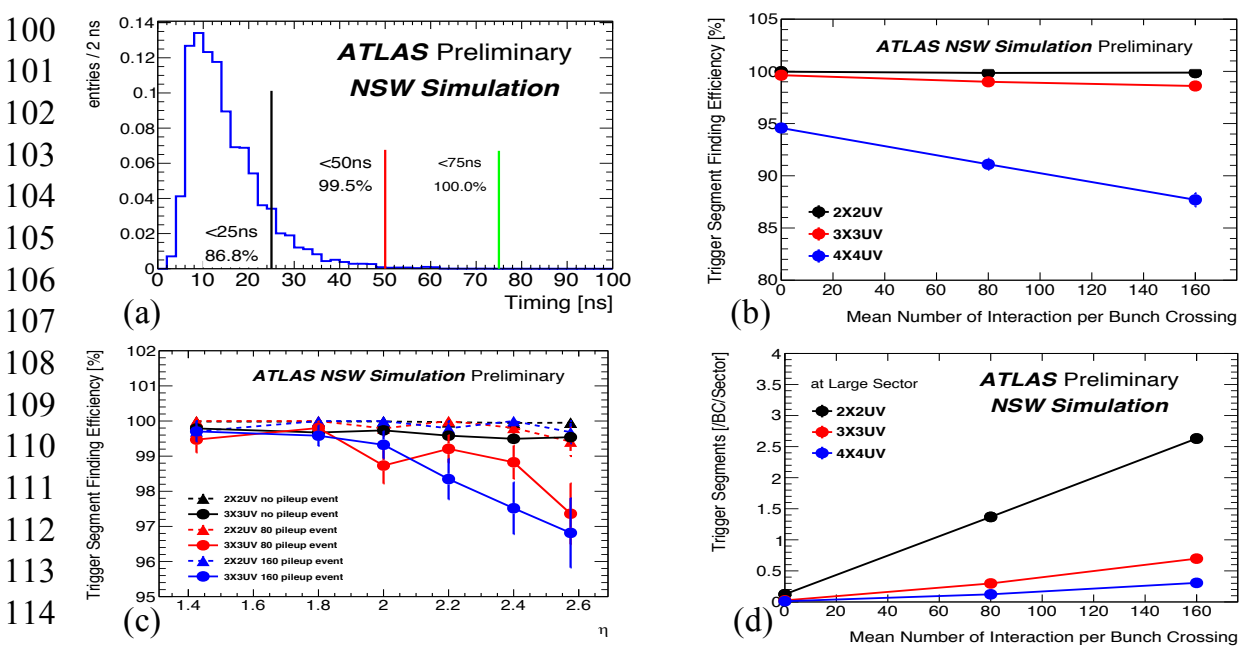

115 Fig. 3 (a) Timing distribution of ART output. (b) Trigger segment finding efficiency as a func116 tion of the number of pileup events. (c) Trigger segment finding efficiency as a function of $\eta$.

117 (d) Average rate of trigger segments per crossing per sector as a function of the number of

118 pileup events.

\section{$119 \quad 5.2 \quad$ Resolution}

120 Resolution of the segment parameters for the 160 pile-up condition is $1.1 \mathrm{mrad}$ for $\Delta \theta$,

$1214.7 \times 10^{-5}$ for $\eta$ and $1.9 \mathrm{mrad}$ for $\phi$. For all the 3 parameters, the resolution meets the 122 requirement.

\section{Conclusion}

124

125

126

127

128

129

The MM trigger performance is studied using a detailed simulation of single muons plus high pile up background. Trigger segment finding efficiency is found to be higher than $97 \%$ even in the highest hit rate conditions. The segment resolution meets the requirement and trigger link occupancy is low. The study shows the MM trigger maintains its performance in the highest background rate condition for the future LHC operation.

\section{References}

1. Evans, L., Bryant, P.: LHC Machine. JINST 3 S08001 (2008).

2. ATLAS Collaboration: The ATLAS Experiment at the CERN Large Hadron Collider. JINST 3 S08003 (2008).

3. ATLAS Collaboration: New Small Wheel Technical Design Report. CERN-LHCC-2013006. CERN, Geneva (2013).

4. Rossi, L., Brüning, O.: High Luminosity Large Hadron Collider A description for the European Strategy Preparatory Group. CERN-ATS-2012-236. CERN, Geneva (2012). 